\title{
Comparing costs and health care resource utilization between nmHSPC and mHSPC patients: a retrospective claims analysis
}

Gilbert Chao Ko, PharmD, MBA, MS; Ryan Hansen, PharmD, PhD; and Josh Carlson, PharmD, PhD

\section{What is already known about this subject}

- Studies have been conducted comparing the costs and health care resource utilization (HCRU) between nonmetastatic castrationresistant prostate cancer ( $\mathrm{nmCRPC}$ ) and metastatic castration-resistant prostate cancer (mCRPC) patients, but not in the hormone-sensitive prostate cancer (HSPC) setting.

- Many new treatments have been approved for $\mathrm{mHSPC}$, with several more in the pipeline, adding to the expansive options available to patients.

- The expansion of available treatments will provide patients with additional options but may also increase costs and HCRU to the health care system.

\section{What this study adds}

- This study presents the incremental costs and HCRU that patients with mHSPC incur compared to patients with nmHSPC.

- Study findings can be used to identify financial burdens in patient subgroups and inform cost-effectiveness models in the HSPC space to assess the value of novel treatments.

- To date, this is the first populationlevel analysis of the incremental costs and HCRU of patients with $\mathrm{mHSPC}$ compared to patients with nmHSPC.

\section{ABSTRACT}

BACKGROUND: Prostate cancer is common among men in the United States, and hormone sensitive-prostate cancer (HSPC) is the predominant etiology. However, there is a paucity of evidence documenting the financial impact of metastatic disease within this etiology.

OBJECTIVE: To estimate the differences in health care resource utilization (HCRU) and costs for patients with nonmetastatic HSPC (nmHSPC) and metastatic HSPC (mHSPC) and their payers.
METHODS: We conducted a retrospective cohort analysis using claims data from the IBM MarketScan databases from January 2016 to December 2019. HSPC was defined as having at least 1 inpatient services or 2 outpatient services claims with a prostate cancer diagnosis and a claim for androgen deprivation therapy use within 6 months of the initial diagnostic claim. Metastatic patients had a secondary diagnosis code of metastasis with their initial diagnostic claim. We compared mean 12-month HCRU, patient out-of-pocket (OOP), and payer costs between patients with nmHSPC and $\mathrm{mHSPC}$ using multivariable linear regression.

\section{Author affiliations \\ Gilbert Chao Ko, PharmD, MBA, MS; Ryan Hansen, PharmD, PhD; and Josh Carlson, PharmD, PhD, Department of Pharmacy, University of Washington, Seattle.}

\section{AUTHOR CORRESPONDENCE:}

Gilbert Chao Ko, 206.397.6142;

gilbko@uw.edu

J Manag Care Spec Pharm. 2022;28(3):287-95

Copyright $\odot 2022$, Academy of Managed Care Pharmacy. All rights reserved.

RESULTS: 4,329 patients met the study inclusion criteria, 600 of which had mHSPC. Patients with $\mathrm{mHSPC}$ had more outpatient prescription fills (10.91 additional fills; 95\% $\mathrm{Cl}=8.09-13.99)$ and outpatient services visits (10.61 additional visits; $95 \% \mathrm{Cl}=8.38$ 13.04) but similar other HCRU. The overall 12-month costs of patients with MHSPC were significantly greater than patients with nmHSPC for patient $\operatorname{OOP}(\$ 1,244$; $95 \% \mathrm{Cl}=\$ 1,004-\$ 1,513)$ and payers $(\$ 113,725$; $95 \% \mathrm{Cl}=\$ 91,494-\$ 141,127)$.

CONCLUSIONS: Compared with patients with nmHSPC, individuals with $\mathrm{mHSPC}$ incur greater HCRU and significant personal and overall financial burden. 
Prostate cancer is the most commonly diagnosed cancer among men in the United States and is the second leading cause of cancer death in American men. ${ }^{1}$ It is estimated that in 2020 there were more than 200,000 new cases of prostate cancer in the United States, with over 30,000 deaths attributed to the disease. ${ }^{1}$ Approximately 1 in 9 men will be diagnosed with prostate cancer during their lifetime, with approximately $60 \%$ of cases diagnosed in men over age $65 .{ }^{1}$ The economic burden of prostate cancer is high, with major contributing factors such as high prevalence and the long duration of the disease. A 2010 study by the National Cancer Institute estimated the US costs associated with prostate cancer to be $\$ 11.85$ billion; the burden of prostate cancer has only increased in the decade since because of the rising prevalence of the disease. ${ }^{2}$ The Global Cancer Observatory, part of the World Health Organization, has predicted that the number of incident cases of prostate cancer will rise by $33.6 \%$ between 2018 and $2040 .^{3}$

The majority of men newly diagnosed with prostate cancer have localized hormone-sensitive disease, which can be complicated by progression to metastatic or castration-resistant disease, or both. ${ }^{4}$ The pathophysiology of castration-resistant disease is unclear, although it has been suggested that development of androgen-independent cells may be a contributing factor. ${ }^{5}$ Although prognosis is poor, only $10 \%-20 \%$ of men diagnosed with prostate cancer will develop castration-resistant disease.$^{5}$ The majority of the burden of prostate cancer still remains with hormone-sensitive prostate cancer (HSPC). Prevalence of nonmetastatic hormone-sensitive prostate cancer (nmHSPC) is estimated at approximately 73.6 cases per 100,000, compared with 9.4 cases per 100,000 for metastatic hormone-sensitive prostate cancer (mHSPC). ${ }^{6}$ According to Surveillance, Epidemiology, and End Results (SEER) data from 2010 to 2016, nmHSPC has a 5-year relative survival rate of $100 \%$, whereas mHSPC has a dismal $30 \%{ }^{6}$ Despite the high relative survival rate of nmHSPC, the long duration of the disease can be a substantial burden in terms of costs and health care resource utilization (HCRU).

Curative treatment options for localized HSPC generally consists of surgery or radiotherapy. ${ }^{7}$ Androgen deprivation therapy (ADT) is the primary systemic therapy for regional or advanced HSPC. ${ }^{8}$ ADT consists of orchiectomy, a luteinizing hormone-releasing hormone (LHRH) agonist or antagonist, or an LHRH agonist plus a first-generation antiandrogen. In patients who present with mHSPC, ADT is the gold standard for initial treatment. ${ }^{8}$ Docetaxel provides an additional treatment option in conjunction with ADT. ${ }^{8}$ Novel hormonal therapies such as apalutamide, abiraterone, and enzalutamide are newly indicated treatments for mHSPC, after a spate of recent approvals by the
US Food and Drug Administration. ${ }^{8}$ Treatment for HSPC has advanced greatly in the past 2 decades, improving the treatment paradigm for patients. However, the growth of available treatment options for HSPC has driven an increase in its economic impact. ${ }^{9}$

Although a multitude of studies have been published on the economic burden of prostate cancer, there is an evidence gap in terms of recent publications that use real-world data as opposed to predictive modeling and within HSPC specifically. ${ }^{2,9,10}$ There is an even greater paucity of research assessing the differences in economic burden between nmHSPC and mHSPC. Studies have been conducted on the costs and HCRU of castration-resistant prostate cancer, ${ }^{11}$ and in prostate cancer in particular, ${ }^{12,13}$ but there has been a lack of focus on HSPC. A better understanding of these differences would aid in optimizing resource allocation and access and provide useful data for informing health economic analysis as potential treatments for HSPC are investigated.

In this study, we sought to compare the resource utilization and costs among patients with nmHSPC vs mHSPC. Our primary objective was to compare the mean annual HCRU, direct payer, and patient out-of-pocket (OOP) costs between nmHSPC and mHSPC patients in the commercially insured US population and Medicare supplemental beneficiaries. Our secondary objectives were to compare the drivers of resource use and costs by individually evaluating the inpatient and outpatient medical services and outpatient prescription fills between nmHSPC and mHSPC.

\section{Methods}

\section{STUDY DESIGN AND DATA SOURCE}

We used the IBM MarketScan Commercial Claims and Encounters (CCAE) and Medicare Supplemental and Coordination of Benefits (MDCR) databases between January 1, 2016, and December 31, 2019. The CCAE database consists of medical and drug data from employers and health plans and is representative of commercially insured patients. The MDCR database consists of claims data of retirees with Medicare supplemental insurance paid by employers. All patient-level data are deidentified in accordance with the Health Insurance Portability and Accountability Act of 1996 (HIPAA); therefore, the study was deemed not human subjects research by the Human Subjects Division at the University of Washington by self-determination.

\section{SAMPLE SELECTION}

Sample enrollment criteria and timeline are shown in Figure 1. The nmHSPC and mHSPC cohorts included male 


\section{FIGURE 1 Study Enrollment Criteria}

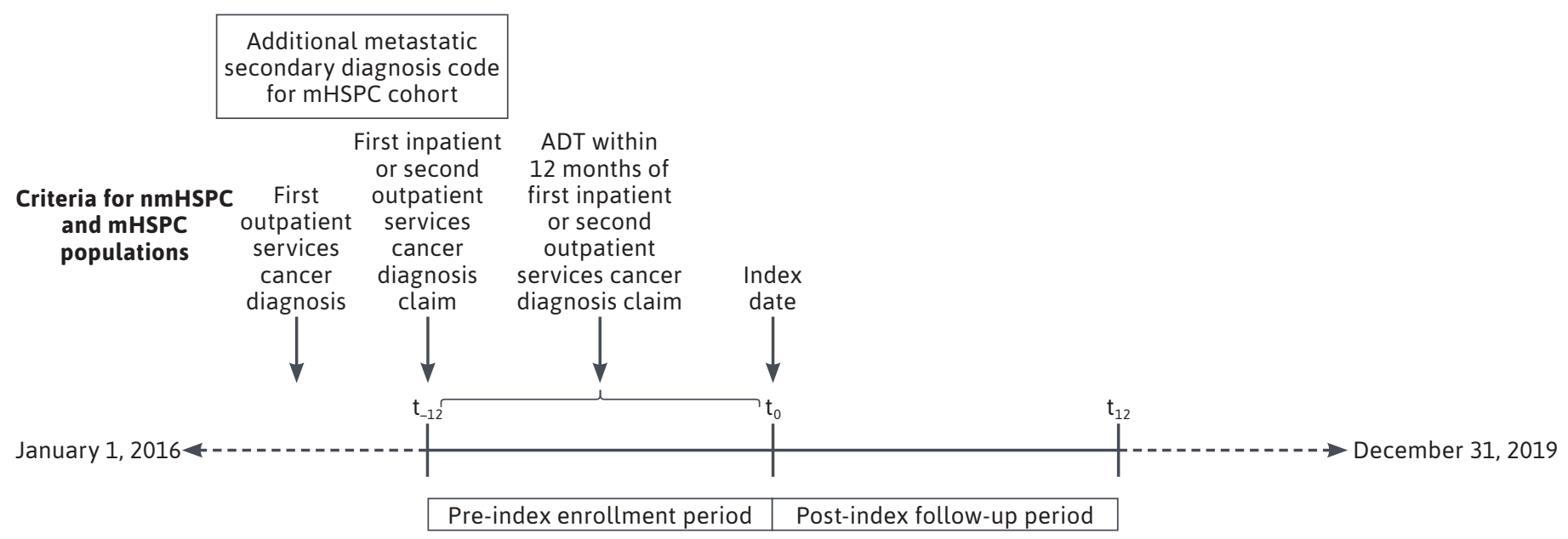

$A D T=$ androgen deprivation therapy; $\mathrm{mHSPC}=$ metastatic hormone-sensitive prostate cancer; $n m H S P C=$ nonmetastatic hormone-sensitive prostate cancer

adult individuals who were aged at least 18 years at the index date, with at least 1 inpatient service or 2 outpatient services claims within a 6-month period that had an International Classification of Diseases, Tenth Revision, Clinical Modification (ICD-10-CM) code of prostate cancer in the primary or secondary diagnosis variable (DX1 or DX2). An individual's first inpatient service or second outpatient service claim with a primary diagnosis of prostate cancer was defined as the diagnostic claim. We required subjects to be continuously enrolled for 24 months from their diagnostic claims. Additionally, individuals must have had at least 1 outpatient services or outpatient pharmaceutical claim for ADT within 12 months of their diagnostic claims. Individuals in the mHSPC cohort must have had an additional diagnosis code for metastasis in their diagnostic claims (DX1-4). Those who were originally nonmetastatic but had any claims with a metastatic diagnosis or treatments for mHSPC (docetaxel, abiraterone, enzalutamide, or apalutamide) between their first prostate cancer claims and end of follow-up were excluded. Diagnosis codes used for inclusion and exclusion are shown in Supplementary Tables 1 and 2, respectively (available in online article).

The index date for each individual was defined as 12 months after the diagnostic claim. This period was chosen to ensure that patients had established disease by the start of the follow-up period and that initial diagnostic and treatment costs were not inadvertently captured. We excluded diagnostic and treatment costs due to the possibility of confounding our results. Because prostate cancer is generally a disease that patients live with over a longer time period than other cancers, excluding the upfront costs provided a more accurate picture of the economic burden of the disease over a patient's lifetime. Individuals were followed for 12 months starting from the index date. Index dates ranged between January 1, 2017, and December 31, 2018, with follow-up ending December 31, 2019.

\section{STUDY MEASURES AND OUTCOMES}

Baseline demographic characteristics, including age, Charlson Comorbidity Index (CCI) score, health insurance plan type, and geographic region were assessed during the pre-index enrollment period. The CCI variable excluded any cancer-related measures because prostate cancer was an inclusion criterion; inclusion of these measures may have inadvertently biased the results of the analysis.

Costs for patient and payers were derived from outpatient drug, outpatient services, and inpatient services claims and summed at the individual level. Patient OOP costs were defined as the sum of coinsurance, copayments, and deductibles. Payer costs were calculated from the net payment variable of each claim. To quantify the contribution that each resource type (inpatient services, outpatient services, outpatient prescription drugs) made to the overall differences in costs, the incremental differences in mean annual cost between the nmHSPC and mHSPC cohorts were also calculated by resource type. All costs were reported in 2020 US dollars, with the previous years' costs 


\section{FIGURE 2 Sample Attrition}

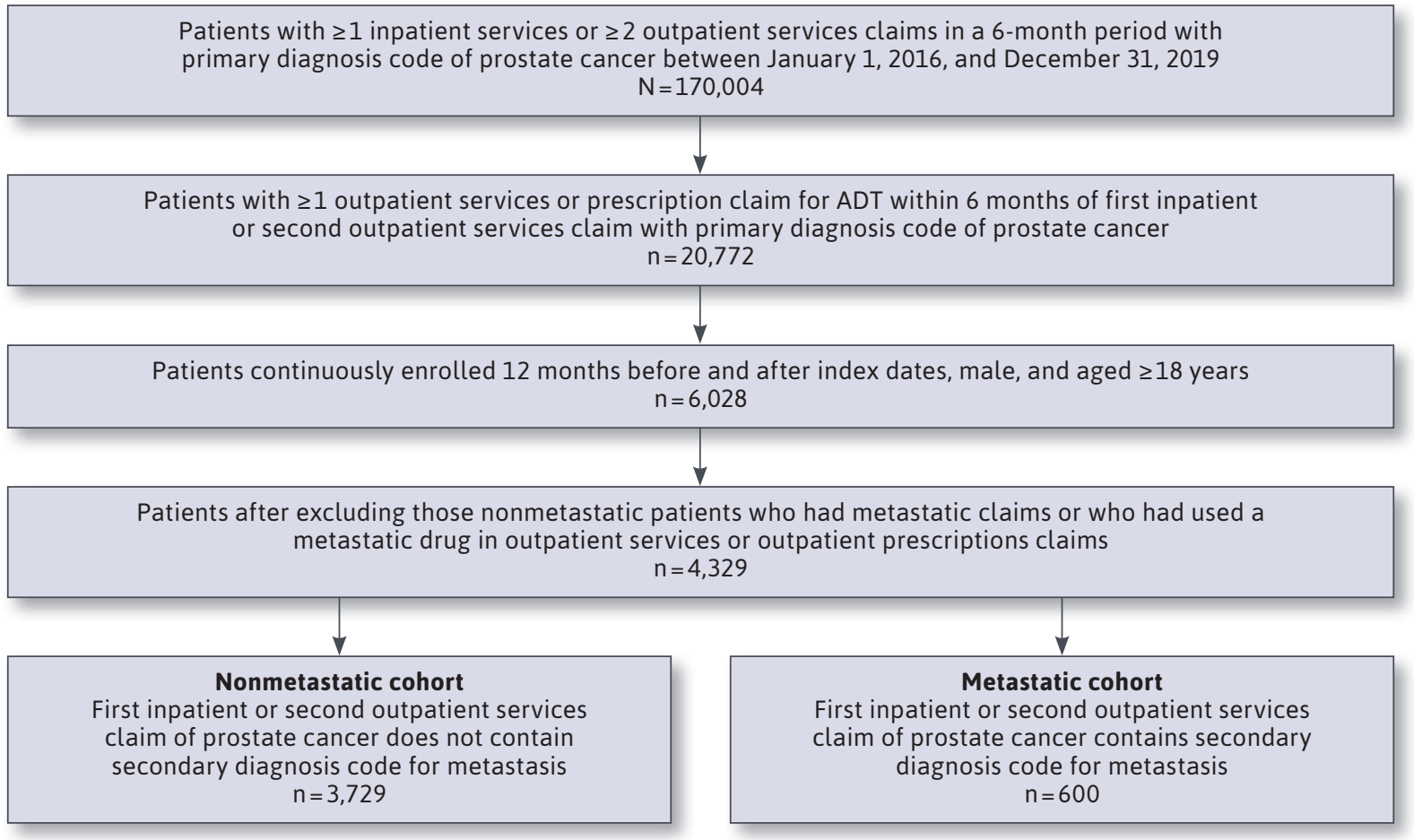

$A D T=$ androgen deprivation therapy

inflated using the medical care component of the Consumer Price Index.

HCRU was estimated by counting days spent in inpatient services, unique outpatient services visits, and number of outpatient drug fills. Unique outpatient visits was chosen as the outcome instead of days spent in outpatient services because a patient could have multiple visits for different outpatient services in a single day. HCRU data were sourced from inpatient services, outpatient services, and outpatient drug claims. The MarketScan variable Quantity of Services (QTY) was summed for each individual to obtain number of outpatient drug fills, in order to take into account transaction reversals that are not uncommon in claims data. Selecting claims with only positive QTY and excluding claims with negative QTY would falsely inflate the apparent number of outpatient drug claims for individuals.

\section{STATISTICAL ANALYSIS}

Baseline demographic characteristics were summarized, using means and SDs for continuous variables and frequencies and proportions for categorical variables. The Student's t-test was used to compare differences for continuous variables, and chi-square tests were used to assess differences in categorical variables between the nmHSPC and mHSPC cohorts.

Differences in the resource use and cost outcomes between patients with nmHSPC and patients with mHSPC were estimated using generalized linear models with gamma distribution. These models included a binary covariate identifying metastasis and adjusted for age (10-year categories); CCI score $(0,1,2$, and $3+)$; health insurance plan type (comprehensive, exclusive provider organization, health maintenance organization, point of service, preferred provider organization, point of service with capitation, consumer-directed health plan, and high deductible health plan); and geographic region (North Central, Northeast, South, West, and unknown). Separate models were estimated for each outcome and the subtypes of resource use and cost. 


\section{TABLE 1}

Baseline Demographics and Clinical Characteristics of Study Participants

\begin{tabular}{|c|c|c|c|c|}
\hline Characteristic & Nonmetastatic & Metastatic & $P$ value & SMD \\
\hline $\mathbf{n}$ & 3,729 & 600 & & \\
\hline Age, mean (SD) & $68.0 \quad(11.2)$ & $68.5 \quad(11.3)$ & 0.3492 & 0.041 \\
\hline Index year, mean (SD) ${ }^{a}$ & $2017 \quad(0.5)$ & 2017 & $<0.001$ & 0.177 \\
\hline
\end{tabular}

Age group, $n$ (\%)

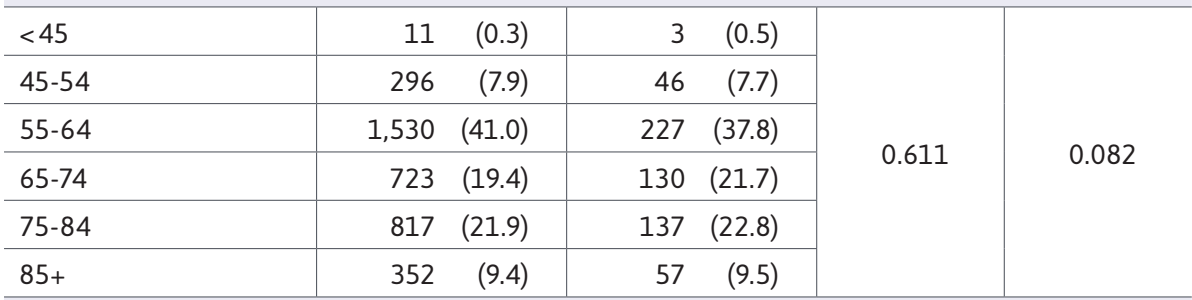

\begin{tabular}{|c|c|c|c|c|}
\hline Region, n (\%) & & & & \\
\hline Northeast & $1,045 \quad(28.0)$ & $186 \quad(31.0)$ & \multirow{5}{*}{0.001} & \multirow{5}{*}{0.185} \\
\hline North Central & $737 \quad(19.8)$ & $135 \quad(22.5)$ & & \\
\hline South & $1,451 \quad(38.9)$ & $183(30.5)$ & & \\
\hline West & $486 \quad(13.0)$ & 92 (15.3) & & \\
\hline Unknown & $10 \quad(0.3)$ & $4 \quad(0.7)$ & & \\
\hline \multicolumn{5}{|c|}{ CCl category, n (\%) } \\
\hline 0 & $1,758 \quad(47.1)$ & $264(44.0)$ & \multirow{4}{*}{0.380} & \multirow{4}{*}{0.076} \\
\hline 1 & $932 \quad(25.0)$ & $160 \quad(26.7)$ & & \\
\hline 2 & $350 \quad(9.4)$ & $57 \quad(9.5)$ & & \\
\hline 3 & 689 (18.5) & 110 (18.3) & & \\
\hline
\end{tabular}

continued on next page

Costs and HCRU were summed for individual patients over their follow-up periods, with zero values excluded in analysis. In order to identify the drivers behind the differences observed in patients with mHSPC and nmHSPC for outpatient prescription fills and services, we analyzed the outpatient prescriptions dataset by assessing counts of unique National Drug Code (NDC) numbers, therapeutic classes, and days' supply. We also determined the 15 highest cost procedure codes in the outpatient services dataset for each cohort.

We constructed generalized linear models with gamma distribution to explore differences in costs between the groups by the age of typical Medicare eligibility (stratifying the population at age 65 ), which used age strata in increments of 5 years and also within region strata, by separately estimating the cost outcomes for the nmHSPC and mHSPC cohorts. Separate models were constructed for subgroup analyses for each stratum. We tested for the significance of an interaction between metastasis and age and metastasis and region, using multiple F-tests.

Patient cohorts were identified in SAS, version 9.4 (SAS Institute Inc.), and baseline demographics testing and regression analyses were conducted in $\mathrm{R}$ studio, version 1.4.1106
(Rstudio Inc.). A 2-sided alpha with a significance level of 5\% was used for all statistical comparisons.

\section{Results}

From the inpatient services and outpatient services tables of the MarketScan CCAE and MDCR databases, 4,329 patients were identified who met the prespecified sample selection criteria and were included in the analysis (Figure 2). Of these patients, 3,729 (86.1\%) were in the nmHSPC cohort, and 600 (13.9\%) were in the mHSPC cohort. Baseline demographics and clinical characteristics of patients are shown in Table 1. The average age distribution within age categories was similar between the nmHSPC cohort (mean $[\mathrm{SD}]=68.0 \quad[11.2]$ years) and the mHSPC cohort (mean $[\mathrm{SD}]=68.5$ [11.3] years). The nmHSPC cohort had a higher proportion of patients with a CCI score of 0 compared with the mHSPC cohort $(47.1 \%$ vs $44.0 \%)$, and the remaining distributions were fairly similar for CCI scores of 1,2, and 3 or greater.

The distribution of region was slightly different, with the largest difference in proportions in the South, which contained $38.9 \%$ of the nmHSPC cohort compared with $30.5 \%$ of the mHSPC cohort. None of these differences were statistically significant when evaluated with chi-square tests. In both cohorts, the majority of patients were enrolled in preferred provider plans, did not have full-time employment status, and were not in a labor union, with similar proportions across all categories of these baseline demographics.

The differences in mean patient OOP and payer costs between the nmHSPC and mHSPC cohorts are shown in Table 2. The mean adjusted annual patient OOP costs were significantly greater among patients 


\section{TABLE 1 Baseline Demographics and Clinical Characteristics of Study Participants (continued)}

\begin{tabular}{|c|c|c|c|c|}
\hline Characteristic & Nonmetastatic & Metastatic & $P$ value & SMD \\
\hline \multicolumn{5}{|l|}{ Plan type, n (\%) } \\
\hline Comprehensive & $557 \quad(15.2)$ & $105(18.0)$ & \multirow{8}{*}{0.081} & \multirow{8}{*}{0.166} \\
\hline EPO & $36 \quad(1.0)$ & $7 \quad(1.2)$ & & \\
\hline $\mathrm{HMO}$ & $502 \quad(13.7)$ & $95 \quad(16.3)$ & & \\
\hline POS & $86 \quad(2.4)$ & $20 \quad(3.4)$ & & \\
\hline PPO & $2,008 \quad(54.9)$ & $304 \quad(52.1)$ & & \\
\hline POS with capitation & $135 \quad(3.7)$ & (1.4) & & \\
\hline $\mathrm{CDHP}$ & $207 \quad(5.7)$ & $31 \quad(5.3)$ & & \\
\hline HDHP & $(3.5)$ & $(2.2)$ & & \\
\hline \multicolumn{5}{|l|}{ Union status, n (\%) } \\
\hline Nonunion & $1,798 \quad(48.2)$ & $306 \quad(51.0)$ & \multirow{3}{*}{0.415} & \multirow{3}{*}{0.058} \\
\hline Union & $771 \quad(20.7)$ & $121(20.2)$ & & \\
\hline Other/unknown & $1,160 \quad(31.1)$ & $173(28.8)$ & & \\
\hline \multicolumn{5}{|l|}{ Employment status } \\
\hline Full time & $1,275 \quad(34.2)$ & $193 \quad(32.2)$ & \multirow{2}{*}{0.354} & \multirow{2}{*}{0.043} \\
\hline Not full time & $2,454 \quad(65.8)$ & $407 \quad(67.8)$ & & \\
\hline \multicolumn{5}{|l|}{ Commercial/Medicare } \\
\hline Commercial & $1,836 \quad(49.2)$ & $274 \quad(45.7)$ & \multirow{2}{*}{0.114} & \multirow{2}{*}{0.072} \\
\hline Medicare & $1,893 \quad(50.8)$ & $326 \quad(54.3)$ & & \\
\hline
\end{tabular}

${ }^{a} P<0.001$

$\mathrm{CCl}=$ Charlson Comorbidity Index; $\mathrm{CDHP}=$ consumer-directed health plans; $E P O=$ exclusive provider organization; $H D H P=$ high-deductible health plan; $H M O=$ health maintenance organization; $P O S=$ point of service; $P P O=$ preferred provider organization; $S M D=$ simple mean difference.

in the mHSPC cohort $(\$ 1,244 ; 95 \%$ $\mathrm{CI}=\$ 1,004-\$ 1,513)$, along with mean annual payer costs $(\$ 113,725 ; 95 \%$ $C I=\$ 91,494-\$ 141,127)$.

Outpatient services and outpatient prescriptions appeared to have roughly equal contribution to overall differences between nmHSPC and mHSPC patient OOP costs (outpatient services: \$664; 95\% $\mathrm{CI}=\$ 509-\$ 837 ;$ outpatient prescriptions: $\$ 706 ; 95 \% \mathrm{CI}=\$ 491-\$ 983)$ and payer costs (outpatient services: $\$ 62,657 ; \quad 95 \% \quad C I=\$ 48,857-\$ 80,092$ outpatient prescriptions: $\$ 52,469$; 95\% CI $=\$ 37,322-\$ 74,490$ ).

In the under 65 years subgroup, the mHSPC cohort observed greater annual patient OOP costs (\$2,042; 95\% $\mathrm{CI}=\$ 1,547-\$ 2,626)$ and annual payer costs $(\$ 120,772 ; \quad 95 \% \quad C I=\$ 91,260-$ $\$ 160,577)$. The 65 years and older subgroup had similar results as the younger age subgroup, with the mHSPC cohort observing greater mean annual patient OOP costs $(\$ 647 ; 95 \% \mathrm{CI}=\$ 433-\$ 896)$ and mean annual payer costs $(\$ 87,059 ; 95 \%$ $\mathrm{CI}=\$ 60,275-\$ 123,147)$. The magnitude of difference in mean annual costs between the nmHSPC and mHSPC cohorts appears to be less in the 65 years and older subgroup compared with the younger subgroup. Multiple F-testing showed that age was a significant effect modifier for patient
$(P=0.03)$ and payer $(P<0.01)$ costs, although metastatic patients in both subgroups had higher mean annual patient OOP and payer costs.

In geographic region subgroup analysis, the mHSPC cohort had higher mean annual patient OOP and payer costs in all regions. The South geographical region had the most pronounced differences in costs for mean annual patient OOP costs $(\$ 1,884 ; 95 \% \mathrm{CI}=\$ 1,299-\$ 2,613)$, whereas the Northeast geographical region observed the largest differences in mean annual payer costs (\$135,061; 95\% CI $=\$ 94,425-\$ 195,116)$. The Northeast geographical region had the smallest difference between the nmHSPC and mHSPC cohorts for mean annual patient OOP costs (\$370; 95\% CI $=\$ 160-\$ 619)$, whereas the West geographical region had the least differences in mean annual payer costs $\quad(\$ 74,256 ; \quad 95 \% \quad C I=\$ 43,005-$ $\$ 124,262)$ between cohorts. Multiple F-tests for region for patient OOP was statistically significant $(P=0.01)$.

The difference between the nmHSPC and mHSPC cohorts in terms of mean annual number of days spent in inpatient services, number of unique outpatient services visits, and number of outpatient prescription fills are shown in Table 3. On average, metastatic patients spent a similar number of days in inpatient services compared with nonmetastatic patients $(-0.38$ days; 95\% $\mathrm{CI}=-2.34-2.42)$. Patients in the mHSPC cohort had a higher mean annual number of outpatient services visits compared with the nmHSPC cohort (10.61 visits; 95\% CI=8.38-13.04) and higher mean annual number of outpatient prescription fills compared with nonmetastatic patients (10.91 fills; 95\% CI=8.09-13.99). On average, mHSPC prescription fills consisted of 


\begin{tabular}{|c|c|c|c|c|}
\hline \multirow[b]{2}{*}{ Base case model } & \multicolumn{2}{|c|}{$\begin{array}{l}\text { mHSPC incremental mean } \\
\text { patient OOP costs }(95 \% \mathrm{CI}), \$\end{array}$} & \multicolumn{2}{|c|}{$\begin{array}{l}\text { mHSPC incremental mean } \\
\text { payer costs }(95 \% \mathrm{CI}), \$\end{array}$} \\
\hline & 1,244 & $(1,004-1,513)^{a}$ & 113,725 & $(91,494-141,127)^{\mathrm{a}}$ \\
\hline Inpatient services & -39 & $(-288-327)$ & 12,158 & $(-13,011-52,183)$ \\
\hline Outpatient services & 664 & $(509-837)^{a}$ & 62,657 & $(48,857-80,092)^{a}$ \\
\hline Outpatient prescriptions & 706 & $(491-983)^{a}$ & 52,469 & $(37,322-74,490)^{a}$ \\
\hline \multicolumn{5}{|l|}{ Age subgroup model ${ }^{b}$} \\
\hline$<65$ years & 2,042 & $(1,547-2,626)^{a}$ & 120,772 & $(91,260-160,577)^{a}$ \\
\hline$\geq 65$ years & 647 & $(433-896)^{a}$ & 87,059 & $(60,275-123,147)^{a}$ \\
\hline \multicolumn{5}{|l|}{ Region subgroup modelc } \\
\hline Northeast & 370 & $(160-619)^{a}$ & 135,061 & $(94,425-195,116)^{\mathrm{a}}$ \\
\hline North Central & 1,614 & $(1,079-2,279)^{a}$ & 89,484 & $(55,505-143,058)^{a}$ \\
\hline South & 1,884 & $(1,299-2,613)^{\mathrm{a}}$ & 123,734 & $(86,561-177,158)^{a}$ \\
\hline West & 1,322 & $(816-1,966)^{a}$ & 74,256 & $(43,005-124,262)^{a}$ \\
\hline \multicolumn{5}{|c|}{$\begin{array}{l}\text { Notes: Payer costs were identified using the net payment variable. Patient costs were calculated as the sum } \\
\text { of each individual's copay, coinsurance, and deductible. Models were adjusted for age, CCI score, region, } \\
\text { and insurance plan type unless otherwise noted. } \\
\text { aP }{ }^{a}<0.001 \text {. }\end{array}$} \\
\hline
\end{tabular}

more unique NDC numbers (13.5 vs 11.6), unique therapeutic classes (8.9 vs 7.9), and lower days' supply (45 days vs 51 days).

Identification of the top 15 most expensive outpatient services for each cohort showed no remarkable differences (Supplementary Tables 3 and 4 , available in online article). The most expensive services for patients were not cancer related in the nmHSPC cohort, whereas targeted genomicsequencing panels for solid organ neoplasms and radiosurgery appeared to be the most expensive oncologyrelated services for the $\mathrm{mHSPC}$ cohort. The highest cost oncology-related services for payers in the nmHSPC cohort were sipuleucel-t and cabazitaxel, but for the mHSPC cohort, they were samarium, sipuleucel-t, radium, and radiosurgery.

\section{Discussion}

In this study, we used the IBM MarketScan Commercial and Medicare claims databases to evaluate the mean costs and resource use for patients with MHSPC compared with patients with nmHSPC over a 12-month period. Patients were identified based on claims with a primary or secondary ICD-10-CM diagnosis code for prostate cancer, with at least 1 following claim for ADT to confirm hormonesensitive cancer. Patients with at least 1 claim with a secondary ICD-10-CM code for metastasis were enrolled in the mHSPC cohort. Multivariable linear regression indicated that patient OOP and payer costs were significantly higher for the mHSPC cohort over a 12-month period. Mean annual outpatient services unique visits and prescription fills were significantly higher for the mHSPC cohort. We did not find statistically significant differences for mean annual days spent in inpatient services, supporting our findings that inpatient costs did not contribute meaningfully to the overall differences in costs between the nmHSPC and mHSPC cohorts.

Outpatient services and outpatient prescription claims contributed the most to the cost differences between patients with mHSPC and patients with nmHSPC. This finding highlights outpatient treatment and drugs as the primary drivers in the extra costs observed for metastatic patients and identifies potential opportunities for financial support for patients and cost savings for payers. Although there was a higher proportion of patients with mHSPC who had an inpatient services claim, $22.5 \%$ compared with $12.2 \%$ for the nmHSPC cohort, we did not find a significant effect on annual mean costs of hospitalization.

Interestingly, patients under the age of 65 years appeared to have increased costs and HCRU compared with patients 65 years and older. Subgroup analysis for age indicated that although mean annual patient and payer costs were higher for patients with mHSPC for both age groups, the difference was greater for patients under age 65 years. Similar to the trends observed in costs, the differences were greater in the subgroup under age 65 years. The incremental differences in the subgroup under age 65 years were more than twice as great as the subgroup 65 years and older for both outcomes, highlighting the greater resource utilization of the younger subgroup.

These findings could be because of the slower nature of prostate cancer relative to many other solid organ cancers, where intensive interventions may not be recommended in older patients. Additionally, commercial payers generally offer higher 


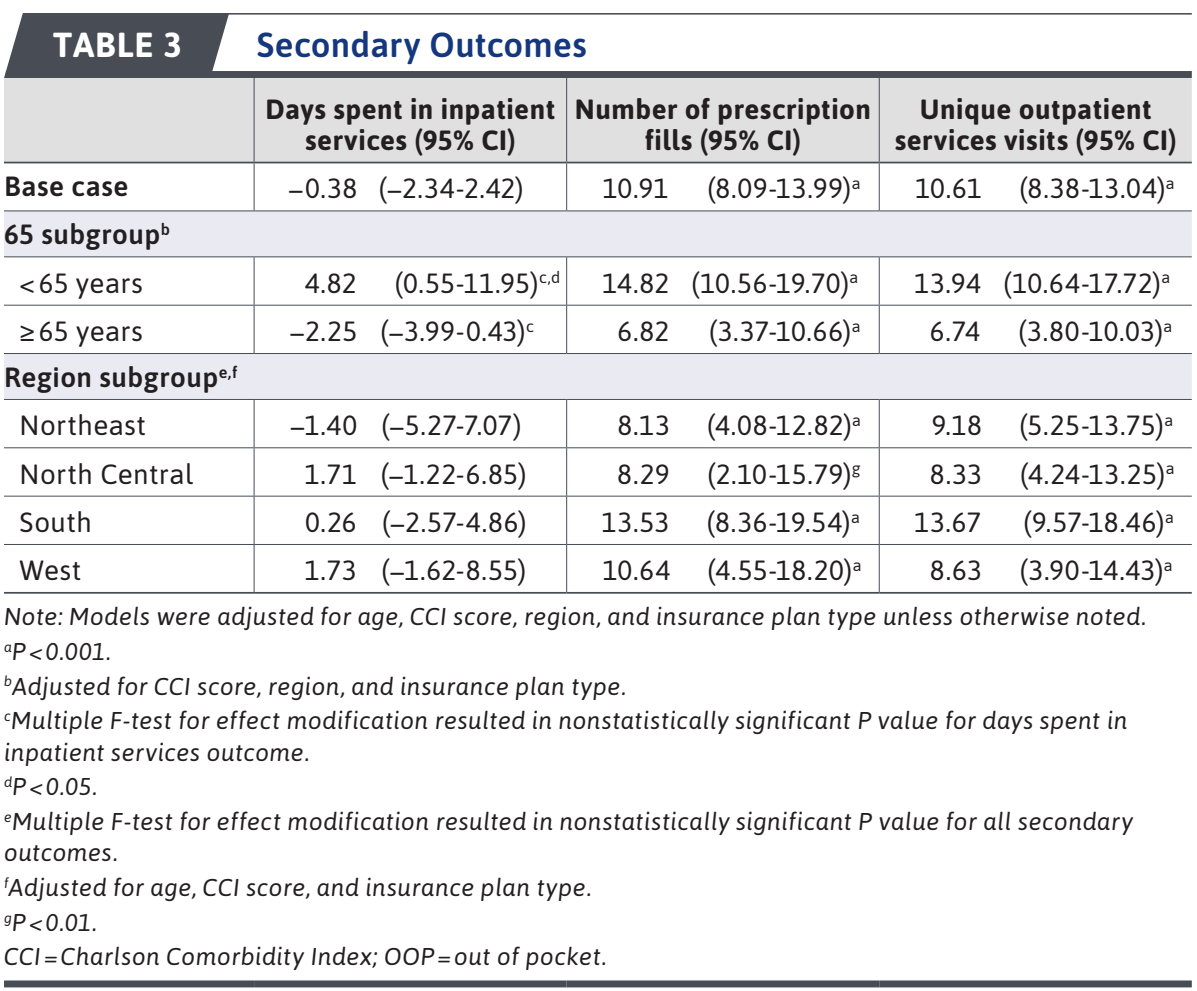

reimbursement rates than Medicare for the same services, which could also have driven the higher costs observed for patients under the Medicare eligibility age. ${ }^{14}$ Palliative care, which would be less expensive than curative treatments, may be used more commonly in older patients.

Overall, patients with mHSPC appear to incur significantly greater costs and health care resources compared with patients with nmHSPC. Analogous trends were observed in several studies that analyzed differences in costs and HCRU, although none focused on HSPC specifically. A retrospective claims analysis that compared differences in costs and HCRU between nonmetastatic and metastatic patients with castrationresistant prostate cancer found an increase of approximately $\$ 120,000$ in mean per-patient-per-year health care costs after patients progressed to metastatic cancer. ${ }^{11}$
HCRU in terms of inpatient admissions and emergency department visits was also observed to have increased in metastatic patients. A retrospective cohort study using data from the SEER cancer registry reported monthly total health care costs of $\$ 2,746$ for localized prostate cancer patients and $\$ 4,677$ for metastatic prostate cancer patients. ${ }^{12}$ This difference equates to $\$ 23,177$ over a 12 -month period, which is lower than the differences observed in our analysis. However, this study did not include the cost of outpatient prescription drugs, which had contributed to a significant portion of the cost differences in our analysis. Similar to our findings, this study found that HCRU was significantly greater for metastatic patients, although different endpoints were used to assess this outcome.

Finally, a more recent study by Tangirala et al found that inpatient and outpatient admissions in metastatic prostate cancer patients was statistically significantly higher in terms of costs than those for nonmetastatic patients ${ }_{13}^{13}$ which aligns with our findings when our results were broken down into inpatient and outpatient services components. Mean annualized costs were not assessed in this study, making it difficult to directly compare the results to our findings, although the general trends in costs align with our conclusions.

\section{LIMITATIONS}

This analysis has several limitations. First, the MarketScan Medicare Supplemental dataset that contributed to the data used in this analysis is not completely representative of the Medicare population. The Medicare Supplemental dataset is derived from MediGap claims, representing the health care experience of retirees with Medicare supplemental insurance paid by employers. All MediGap plans cover Medicare Part A coinsurance and hospital costs, whereas most cover Part A deductible, Part B copays, and coinsurance. The majority of MediGap plans do not cover Part B deductibles, and none cover prescription drugs. This could affect the generalizability of the results for those who are aged 65 years and older. Commercial claims make up the other portion of our dataset, which leaves the Medicaid population unrepresented.

A second limitation is the lack of consistency in ICD-10-CM coding for nmHSPC and mHSPC. Although ICD-10-CM codes exist for these disease states, in practice patients with these diseases are not coded as such. Despite our best efforts to identify each cohort with the use of prostate cancer ICD-10-CM codes and history of ADT use, it is possible that patients who truly had the disease were missed during enrollment or miscoded. 
A third limitation is the continuous enrollment requirement of our study, which only included patients who had health coverage for the 24 months from their first diagnostic claim until the end of their follow-up period. This requirement excluded approximately 14,000 individuals from the analysis and could affect our results.

Finally, MarketScan does not contain variables that could be potential confounders, such as race and socioeconomic status. Therefore, we cannot rule out residual confounding in our results.

\section{Conclusions}

This retrospective claims analysis found that not only do patients with mHSPC pay significantly more than patients with nmHSPC, but payers (employers and insurers) are also affected. Metastatic patients use more health care resources than nonmetastatic patients in terms of outpatient prescription fills and unique outpatient services visits. The cost and HCRU differences between patients with nmHSPC and patients with mHSPC were greater in magnitude for patients under 65 years compared with those who were eligible for Medicare. New agents approved in this space may offer opportunities to delay the development of metastatic disease yet can result in increases in HCRU and costs to patients and payers.

To date, this is the first populationlevel analysis of the incremental costs and HCRU of patients with mHSPC compared with patients with nmHSPC. The results of this research could help in informing health economic models in the HSPC space to ascertain the value of novel treatments. Future research may provide additional insight into benefits of delaying metastatic disease progression by using datasets more inclusive of Medicare and Medicaid populations.

\section{DISCLOSURES}

This study had no outside funding support. The authors have nothing to disclose.

\section{REFERENCES}

1. American Cancer Society. Key statistics for prostate cancer. Updated January 12, 2021. Accessed March 29, 2021. https:// www.cancer.org/cancer/prostate-cancer/about/key-statistics.html

2. Mariotto AB, Yabroff KR, Shao Y, Feuer EJ, Brown ML. Projections of the cost of cancer care in the United States: 2010-2020. J Natl Cancer Inst. 2011;103(2):117-28. doi:10.1093/jnci/djq495

3. Rawla P. Epidemiology of prostate cancer. World J Oncol. 2019;10(2):63-89. doi:10.14740/wjon1191

4. Leslie SW, Soon-Sutton TL, Sajjad H, et al. Prostate cancer. In: StatPearls. Updated May 4, 2021. StatPearls Publishing; 2021. Accessed June 8, 2021. https://www.ncbi.nlm.nih.gov/books/ NBK470550/

5. Kirby M, Hirst C, Crawford ED. Characterising the castration-resistant prostate cancer population: a systematic review. Int J Clin Pract. 2011;65(11):1180-92. doi:10.1111/j.1742-1241.2011.02799.x

6. Surveillance, Epidemiology, and End Results Program. Cancer stat facts: prostate cancer. Accessed March 29, 2021. https://seer.cancer.gov/statfacts/html/ prost.html

7. Brawley S, Mohan R, Nein CD. Localized prostate cancer: treatment options. Am Fam Physician. 2018;97(12):798-805.
8. Mohler JL, Antonarakis ES, Armstrong AJ, et al. Prostate cancer, version 2.2019. NCCN Clinical Practice Guidelines in Oncology. J Natl Compr Canc Netw. 2019;17(5):479-505. doi:10.6004/jncen.2019.0023

9. Gustavsen G, Gullet L, Cole D, Lewine N, Bishoff JT. Economic burden of illness associated with localized prostate cancer in the United States. Future Oncol. 2020;16(1):4265-77. doi:10.2217/ fon-2019-0639

10. Roehrborn CG, Black LK. The economic burden of prostate cancer. BJU Int. 2011;108(6):806-13. doi:10.1111/j.1464-410X.2011.10365.X

11. Appukkuttan S, Tangirala K, Babajanyan S, Wen L, Simmons S, Shore N. A retrospective claims analysis of advanced prostate cancer costs and resource use. Pharmacoecon Open. 2020;4(3):439-47. doi:10.1007/s41669-019-00185-8

12. Li TT, Shore ND, Mehra M, et al. Impact of subsequent metastases on costs and medical resource use for prostate cancer patients initially diagnosed with localized disease. Cancer. 2017;123(18):3591-601. doi:10.1002/cncr.30784

13. Tangirala K, Appukkuttan S, Simmons S. Costs and healthcare resource utilization associated with hospital admissions of patients with metastatic or nonmetastatic prostate cancer. Am Health Drug Benefits. 2019;12(6):306-12.

14. Johnson B, Kennedy K, Kurowski D, et al. Comparing commercial and Medicare professional service prices. Health Care Cost Institute. August 13, 2020. Accessed June 1, 2021. https:// healthcostinstitute.org/hcci-research/ comparing-commercial-and-medicareprofessional-service-prices 